various arteries of the male Spontaneously Hypertensive Rat (SHR) - the best animal model for research on human hypertension - after treatment with ENALAPRIL and HYDROCHLOROTHIAZID. Untreated male SHR and Wistar-Kyoto Rats of the same age were used as controls. The PURPOSE of the investigation was to discover:

- to what extent changes in the arterial wall due to hypertension can be reversed by antihypertensive drugs

- whether and to what exten: differences in the reaction to ENALAPRIL HYDROCHLOROTHIAZID exist

- whether changes in the arterial wall due to hypertension may progress to a point at which antihypertensive therapy is no longer effective.

Results: The reactions in the walls of the coronary, basilar, renal, femoral, mesenteric and caudal arteries are not identical, but are specific for the different vessels. The morphological response of the wall is, however, similar, in that the tunica media responds most strongly with a reduction of the pathological enlargement of the intercellular space and by alteration of the ratio of ' $k$ ' to ' $m$ ' smooth muscle cells (STAUBESAND, 1982) in favour of the former, following treatment with either ENALAPRIL or HYDROCHLOROTHIAZID.Semiquantitative morphological examination has also shown that ENALAPRIL offers particularly effective protection against hypertensive damage to the wall of the coronary arteries, and HYDROCHLOROTHIAZID to that of the renal arteries.After the appearance of calcification foci or osmicphilic shrinkage necroses, however, antihypertensive therapy is no longer effective, and these hypitansive changes should therefore be regarded as irreversible.

This research was supported by Messrs. Merck, Sharp and Dohme

P.we.163

\title{
Release of angiotensin I from tissue sites into the systemic and cardiopulmonary circulations
}

\author{
Danser, A.H.J., Koning, M.M., Derkx, F.H.M., Admiraal, P.J.J., Verdouw, P.D. \\ and Schalekamp, M.A.D.H. \\ Departments of Internal Medicine I and Experimental Cardiology, Erasmus University, Rotterdam, The Netherlands
}

Evidence is accumulating that part of plasma angintensin $\mathrm{I}(\mathrm{AI})$ is generated at tissue sites and not by circulating plasma renin. Tc address this issue we studied whole-body AI kinetics and regional AI metabolism and production in the systemic, pulmonary and coronary vascular beds of the pig.

Methods: Highly purified mono-iodinated ${ }^{125} \mathrm{I}$-AI was given via constant infusion into the left cardiac ventricle of 8 captopril-treated and 8 untreated pigs (weight $23-31 \mathrm{~kg}$ ). The use of ${ }^{125} \mathrm{I}-\mathrm{AI}$ for studying the metabolism of endogenous AI was validated with combined infusions of labeled and unlabeled AI. ${ }^{125} \mathrm{I}-\mathrm{AI}$ infusions had no effect on heart rate, blood pressure, cardiac output or coronary blood flow. Steady-state plasma levels of intact ${ }^{125}$ I-AI and ${ }^{125}$ I-AII and endogenous AI and AII were measured, after SepPak extraction and HPLC separation, with gamma counting and RIA, in the aorta, pulmonary artery, right and left atrium and the great cardiac vein. Plasma renin activity (PRA) was also measured at these sampling sites.

Results: Plasma ${ }^{125}$ I-AI decay, which was followed after stopping the infusion until levels $5-10 \%$ of the steady state plas sau had been reached, was mono-exponential with $t_{1 / 2}=0.95 \pm 0.08$ min (mean $\pm S E M$ ) and $0.40 \pm 0.05$ min with and without captopril respectively. Metabolic clearance rates were $5.6 \pm 0.4$ and $14.6 \pm 1.6 \mathrm{~L} / \mathrm{min}$. Distribution volume was equal under both circumstances, $7.7 \pm 0.3$ and $7.5 \pm 0.4 \mathrm{~L}$ (25-30\% of body weight), indicating that $\mathrm{AI}$ is distributed over both plasma and interstitial fluid compartments. Production of extracellular fluid AI (including plasma AI) was $127 \pm 29$ and $47 \pm 11 \mathrm{pmol} / \mathrm{min}$ with and without captopril, whereas AI production by circulating renin was $63 \pm 20$ and $10 \pm 3 \mathrm{pmol} / \mathrm{min}$. The effect of captopril was predominantly $(\mathrm{p}<0.01)$ on the AI production by circulating renin.

${ }^{125}$ I-AI extraction over the the systemic, pulmonary and coronary vascular beds was $57 \pm 4,14 \pm 4$ and $46 \pm 4 \%$ with captopril, and $55 \pm 2,46 \pm 5$ and $52 \pm 5 \%$ without captopril. Despite this rapid extraction the levels of endogenous $\mathrm{AI}$ in the aorta, right atrium and great cardiac vein were not different from each other, both with and without captopril. Withcut captopril, AI was lower in th left atrium than in the pulmonary artery $(p<0.05)$. The time required for PRA to compensate for AI extraction was 22-24, 2-4 and 13-14 sec in the systemic, pulmonary and 
coronary vascular beds with and without captopril. Extraction of plasina $\mathrm{AI}$ and possible release of $\mathrm{AI}$ from tissue into plasma are expected to occur at the level of the capillaries. Since capillary blood trarsit time is only 2-3 sec, net release of AI from tissue sites into the systemic and coronary circulations must have occurred.

Conclusion: A major part of AI present in extracellular fluid is formed at tissue sites and sone of this locally produced AI does reach the plasma compartment. Captopril-induced changes in the production of extracellular fluid AI at tissue sites do not run in parallel with changes in AI production by circulating renin. These findings may have important implications for understanding the mechanism by which drugs interfering with angiotensin formation can affect vascular tone.

P.we.164

\title{
Reduction of blood pressure with calcium supplementation
}

\author{
Sutoo, D., Matsukura, T. and Akiyama, K. \\ Institute of Medical Science, University of Tsukuba, Tsukuba 305, Japan
}

We have previously suggested that calcium ions activate catecholamine synthesizing enzymes through intracerebral calmodulin (Sutoo et al., 1989). On the other hand, it was known that calcium supplements produce a significant reduction of blood pressure in healthy adults, and hypertensive subjects have a reduced concentration of ionized calcium in the serum. We have investigated the role of calcium ions in the regulation of blood pressure in the light of our previous reports and have suggested that central calcium reduces blood pressure through an intracerebral, calmodulin-dependent, catecholamine-synthesizing system (Sutoo et al., 1987). Also, our study suggested that a part of the i.v. injected calcium might transfer to the intracerebral system and reduce blood pressure (Sutoo et al., 1988). The present study was carried out to clarify the effects of continuous oral intake (p.o.) of calcium on the central, catecholamine-dependent, regulation of blood pressure.

The adult male Wistar rats (270-340 g) were divided into 4 groups; the rats were administrated intraventricularly (i.c.v.) and continuously with either $\alpha$-methyltyrosine ( $\alpha$ MPT, inhibitor of tyrosine hydroxylase, $300 \mu \mathrm{g} / 12 \mu \mathrm{l} /$ day) or saline by an osmotic mini-pump (Alza, CA), furthermore each rat group was given freely either tap water or $4 \%(\mathrm{w} / \mathrm{v}$ ) $\mathrm{CaCl}_{2}$ solution. And the changes of the systolic blood pressure (SBP) were compared among rats groups every day by sphygmomanometer (Riken Kaihatsu, Japan). The SBP of rats supplemented orally with calcium dropped significantly from the 9th day compared to that of the control rats. On the other hand, $\alpha$ MPT attenuated the magnitude of calcium-induced hypotensive activity from the 16th day, although $\alpha$ MPT in itself did not cause any significant effects on SBP (Table 1). This study was reconfirmed by the examination of continuous i.p.-load of $\mathrm{CaCl}_{2}$. Namely, SBP of rats administrated i.p. with $\mathrm{CaCl}_{2}(36 \mathrm{mg} /$ day $)$ decreased gradually with the elapse of days, and was significantly changed from the 7th day compared to control level. However, the SBP oi rats continuously administrated i.c.v. with $\alpha$ MPT (300 is $\mu \mathrm{g} /$ day) was not changed by the i.p. administration of $\mathrm{CaCl}_{2}$.

Recentiy, we have reported (Sutoo et al., 1987) that i.c.v. administration of $\mathrm{CaCl}_{2}$ produces a hypotensive response in conscious rats. This effect was abolished by the administration of a calmodulin antagonist or an inhibitor of

Taible 1

Effect of $\alpha$-methyltyrosine on hypotensive response induced by oral intake of $\mathrm{CaCl}_{2}$

\begin{tabular}{|c|c|c|c|c|c|c|c|c|c|}
\hline \multicolumn{2}{|c|}{ Treatment } & \multicolumn{8}{|c|}{ After administration (day) } \\
\hline p.o. & i.c.v. & 1 & 5 & 9 & 11 & 12 & 14 & 16 & 18 \\
\hline $\begin{array}{l}\text { Water } \\
\text { Water } \\
\mathrm{CaCl}_{2} \\
\mathrm{CaCl}_{2}\end{array}$ & $\begin{array}{l}\text { Saline } \\
\alpha \mathrm{MPT} \\
\text { Saline } \\
\alpha \mathrm{MPT}\end{array}$ & $\begin{array}{r}7.9 \pm 4.7 \\
-3.1 \pm 3.8 \\
2.5 \pm 3.4 \\
3.3 \pm 3.1\end{array}$ & $\begin{array}{r}6.0 \pm 4.3 \\
-5.1 \pm 4.1 \\
1.4 \pm 3.3 \\
4.1 \pm 3.4\end{array}$ & $\begin{array}{r}9.2 \pm 6.1 \\
-6.7 \pm 5.2 \\
-19.7 \pm 5.1^{b} \\
-4.8 \pm 4.6\end{array}$ & $\begin{array}{r}9.0 \pm 5.6 \\
-5.7 \pm 5.0 \\
-26.7 \pm 4.9^{b} \\
-13.3 \pm 5.3^{a}\end{array}$ & $\begin{array}{c}6.3 \pm 5.1 \\
-5.8 \pm 4.5 \\
-29.6 \pm 4.2^{b} \\
-25.8 \pm 6.4^{b}\end{array}$ & $\begin{array}{c}10.0 \pm 6.6 \\
-4.4 \pm 4.4 \\
-35.7 \pm 4.2^{b} \\
-28.1 \pm 5.8^{b}\end{array}$ & $\begin{array}{c}7.7 \pm 4.8 \\
-3.3 \pm 3.5 \\
-44.9 \pm 4.0^{b} \\
-31.0 \pm 5.1^{b c}\end{array}$ & $\begin{array}{c}6.8 \pm 4.6 \\
-5.1 \pm 4.4 \\
-50.9 \pm 5.6^{b} \\
-30.1 \pm 5.4^{b d}\end{array}$ \\
\hline
\end{tabular}

Values are means \pm S.E. of 7 experiments. SBP values at each time were subtracted by pre-administration value. ${ }^{\mathrm{a}} \mathrm{P}<0.05,{ }^{\mathrm{b}} \mathrm{P}<0.01$ vs. water + saline; ${ }^{c} \mathrm{P}<0.05{ }^{\mathrm{d}} \mathrm{P}<0.01$ vs. $\mathrm{CaCl}_{2}+$ saline by Newman-Keuls t-test. 